\title{
A New Second Order Numerical Scheme for Solving Forward Backward Stochastic Differential Equations with Jumps
}

\author{
Hongqiang Zhou, Yang Li, Zhe Wang \\ College of Science, University of Shanghai for Science and Technology, Shanghai, China \\ Email: 18021042159@163.com, 136153646@qq.com
}

Received 1 April 2016; accepted 26 July 2016; published 29 July 2016

Copyright (C) 2016 by authors and Scientific Research Publishing Inc.

This work is licensed under the Creative Commons Attribution International License (CC BY). http://creativecommons.org/licenses/by/4.0/

(c) (i) Open Access

\section{Abstract}

In this paper, we propose a new second order numerical scheme for solving backward stochastic differential equations with jumps with the generator $f=r\left(t, x_{t}, y_{t}\right)+h(t) z_{t}+g(t) \Gamma_{t}$ linearly depending on $z_{t}$. And we theoretically prove that the convergence rates of them are of second order for solving $y_{t}$ and of first order for solving $z_{t}$ and $\Gamma_{t}$ in $L^{p}$ norm.

\section{Keywords}

\section{Numerical Scheme, Error Estimates, Backward Stochastic Differential Equations}

\section{Introduction}

Bismut (1973) studied the existence of the linear backward stochastic differential equation, the results could be regarded as a promotion of a famous Girsanov theorem. The existence and uniqueness of solutions for nonlinear backward stochastic differential equations (BSDEs) were first proved by Pardoux and Peng (1990).Since then, BSDEs have been extensively studied by many researchers. In [1], Peng obtained the relation between the backward stochastic differntial equation and the parabolic partial differential equation (PDE), and in Peng (1990), the stochastic maximum principle for optimal control problems were based on BSDEs. The applications of BSDEs now cover many scientific fields, such as stochastic control, stock markets, risk measure, turbulence fluid flow, biology, chemical reactions, partial differential equations, and so on. Thus it is very important and useful to obtain solutions of BSDEs for real applications. However, it is often quite difficult to obtain analytic solutions of BSDEs, so computing approximate solutions of BSDEs become highly desired, by using the relation between the BSDE and PDE. As far as we know, there have been very few schemes obtained with second-order 
convergence rate, such as [2] [3].

In this paper, we propose a new second order numerical scheme for the solution of forward-backward stochastic differential qquations (FBSDE in short) with jumps with the following form

$$
\left\{\begin{array}{l}
x_{t}=x_{0}+\int_{0}^{t} b\left(s, x_{s}\right) \mathrm{d} s+\int_{0}^{t} \sigma\left(s, x_{s}\right) \mathrm{d} W_{s}+\int_{0}^{t} \int_{\mathcal{E}} \beta\left(s, x_{s-}, e\right) \tilde{N}_{k}(\mathrm{~d} s, \mathrm{~d} e) \\
y_{t}=g\left(x_{T}\right)+\int_{t}^{T} f_{s} \mathrm{~d} s-\int_{t}^{T} Z_{s} \mathrm{~d} W_{s}-\int_{t}^{T} \int_{\mathcal{E}} U_{s} \tilde{N}_{k}(\mathrm{~d} s, \mathrm{~d} z)
\end{array}\right.
$$

From [4], we know that the solution $\left(x_{t}, y_{t}, z_{t}, \Gamma_{t}\right)$ can be represented as

$$
\begin{aligned}
& y_{t}=u\left(t, x_{t}\right), \quad z_{t}=\nabla_{x} u\left(t, x_{t}\right) \sigma\left(t, x_{t}\right), \\
& \Gamma_{t}=\int_{\varepsilon}\left\{u\left(t, x_{t}+\beta\left(t, x_{t}, e\right)\right)-u\left(t, x_{t}\right)\right\} \rho(e) \lambda(\mathrm{d} e), \quad t \in[0, T),
\end{aligned}
$$

where the vector function $u(t, x)$ is the classical solution of the following parabolic differential equation (PDE) of the form

$$
\left\{\begin{array}{l}
\mathcal{L} u(t, x)+f\left(t, x, u(t, x), \nabla_{x} u(t, x) \sigma(t, x), \mathcal{I}[u](t, x)\right)=0 \\
u(T, x)=g(x)
\end{array}\right.
$$

where $\nabla_{x} u$ denotes the gradient of $u$ with respect to the space variable $x$,

$$
\begin{aligned}
& \mathcal{L} u(t, x):= \frac{\partial u}{\partial t}(t, x) \nabla_{x} u(t, x) b(x)+\frac{1}{2} \sum_{i, j=1}^{d}\left(\sigma \sigma^{*}(x)\right) \frac{\partial^{2} u}{\partial x^{i} \partial x^{j}}(t, x) \\
&+\int_{\varepsilon} u(t, x+\beta(x, e))-u(t, x)-\nabla_{x} u(t, x) \beta(x, e) \lambda(\mathrm{d} e), \\
& \mathcal{I}[u](t, x):=\int_{\varepsilon}\{u(t, x+\beta(x, e))-u(t, x)\} \rho(e) \lambda(\mathrm{d} e) .
\end{aligned}
$$

\section{Preliminaries and Notation}

Let $T$ be a fixed positive number and $\left\{\Omega, \mathcal{F}, P,\left\{\mathcal{F}_{t}\right\}_{0 \leq t \leq T}\right\}$ be a complete,filtered probability space on which is defined a standard Brownian motion $W_{t}$, such that $\left\{\mathcal{F}_{t}\right\}_{0 \leq t \leq T}$ is the natural filtration of the Brownian motion $W_{t}$ and all the P-null sets are augmented to each $\sigma$-field $\mathcal{F}_{t}$. Denote by $L^{2}$ the set of all $\mathcal{F}_{t}$-adapted and mean-square-integrable processes.

A process $\left(x_{t}, y_{t}, z_{t}, \Gamma_{t}\right):[0, T] \times \Omega \rightarrow R^{m} \times R^{m \times d}$ is called an $L^{2}$-adapted solution of the FBSDE(1) if it's $\left\{\mathcal{F}_{t}\right\}$-adapted and $L^{2}$-integrable, and satisfies (1). Under some standard conditions on the functions $f$ and $h$, there is a unique adapted random process.

Now we introduce a new probability space: for $\left\{\Lambda_{t}: t \geq 0\right\}$ is an exponential martingale and satisfies $\mathbb{E}_{t_{n}}^{x}\left(\Lambda_{t}\right)=1$, we define $\tilde{\mathbb{E}}[X]=\tilde{\mathbb{E}}\left[X \Lambda_{t_{n}}^{t_{n+1}}\right]$. The random processes $\Lambda_{t_{n}}^{t}$, it is easy to verify that $\Lambda_{t_{n}}^{t}$ is an exponential martingale.

$$
\Lambda_{0}^{t}=\exp \left\{\int_{0}^{t} h(s) \mathrm{d} W_{s}-\frac{1}{2} \int_{0}^{t} h^{2}(s) \mathrm{d} s\right\} \prod_{0 \leq s \leq t}\left[1+\int_{\mathcal{E}} g(s) \rho(e) N_{k}(\{s\}, \mathrm{d} e)\right] \mathrm{e}^{-\int_{0}^{t} \int_{\mathcal{E}} g(s) \rho(e) \pi(\mathrm{de}) \mathrm{d} s} .
$$

Let us first introduce the following lemma.

Lemma 1. Given the time partition $0 \leq t_{n} \leq t_{n+1} \leq T, X$ is a $\mathcal{F}_{t}$-measurable random variable,and satisfies $\tilde{\mathbb{E}}[X]<\infty$.

$$
\tilde{\mathbb{E}}_{t_{n}}^{x^{n}}[X]=\frac{\mathbb{E}_{t_{n}}^{x^{n}}\left[X \Lambda_{0}^{t_{n+1}}\right]}{\Lambda_{0}^{t_{n}}}=\mathbb{E}_{t_{n}}^{x^{n}}\left[X \Lambda_{t_{n}}^{t_{n+1}}\right] \text {, a.s. }
$$

We use the following Itô-Taylor approximation to solve the forward SDEs with jumps

$$
X^{n+1}=X^{n}+\sum_{\alpha \in T_{2}} I_{\alpha}\left[f_{\alpha}\left(t_{n}, X^{n}\right)\right]_{t_{n}, t_{n+1}},
$$


where

and the coefficient function

$$
I_{\alpha}[g(\cdot)]_{t_{n}, \tau}=\left\{\begin{array}{l}
g(\tau), l=0, \alpha=v \\
\int_{t_{n}}^{\tau} I_{\alpha-}[g(\cdot)]_{t_{n}, r} \mathrm{~d} r, l \geq 1, j_{l}=0 \\
\int_{t_{n}}^{\tau} I_{\alpha-}[g(\cdot)]_{t_{n}, r} \mathrm{~d} W_{r}^{j_{l}}, l \geq 1, j_{l} \in 1,2, \cdots, m \\
\int_{t_{n}}^{\tau} \int_{\mathcal{E}} I_{\alpha-}[g(\cdot)]_{t_{n}, r-} N(\mathrm{~d} e, \mathrm{~d} r), l \geq 1, j_{l}=-1
\end{array}\right.
$$

with

$$
f_{\alpha}(t, x, e)=\left\{\begin{array}{l}
f(t, x), l(\alpha)=0 \\
L^{j_{1}} f_{-\alpha}(t, x, e), l(\alpha) \geq 1, j_{1} \in 0,1, \cdots, m, \\
L_{e}^{-1} f_{-\alpha}(t, x, e), l(\alpha) \geq 1, j_{l}=-1
\end{array}\right.
$$

$$
\begin{aligned}
& L^{0} f(t, x, e)=\frac{\partial}{\partial t} f(t, x, e)+\sum_{i=1}^{d} a^{i}(t, x) \frac{\partial}{\partial x^{i}} f(t, x, e)+\frac{1}{2} \sum_{i, l=1}^{d} \sum_{j=1}^{m} b^{i, j}(t, x) b^{l, j}(t, x) \frac{\partial^{2}}{\partial x^{i} \partial x^{l}} f(t, x, e), \\
& L^{k} f(t, x, e)=\sum_{i=1}^{d} b^{i, k}(t, x) \frac{\partial}{\partial x^{i}} f(t, x, e), \\
& L_{e}^{-1} f(t, x, e)=f(t, x+c(t, x, v), e)-f(t, x, e), t \in[0, T], x \in R^{d}, e \in \varepsilon^{s} .
\end{aligned}
$$

Now we introduce some basic notations.

- $\left\{\mathcal{F}_{t}\right\}_{t \leq s \leq T}$ : the $\sigma$-field generated by the Brownian motion.

- Throughout this paper, we denote by $C$ a generic constant depending only on $T$, the upper bounds of the derivatives of the functions $f$.

\section{Numerical Schemes for Solving BSDE}

From the time interval $[0, T]$, we introduce the following time partition: $0=t_{0}<\cdots<t_{N}=T$, let $\Delta t_{n}=t_{n+1}-t_{n}$ and $\max _{0 \leq n \leq N-1} \Delta t_{n}=h$. According to (1), it's easy to obtain that for $0 \leq n \leq N-1$,

$$
y_{t_{n}}=y_{t_{n+1}}+\int_{t_{n}}^{t_{n+1}}\left(r\left(s, x_{s}, y_{s}\right)+h(s) z_{s}+g(s) \Gamma_{s}\right) \mathrm{d} s-\int_{t_{n}}^{t_{n+1}} z_{s} \mathrm{~d} W_{s}-\int_{t_{n}}^{t_{n+1}} \int_{\mathcal{E}} U_{s} \tilde{N}_{k}(\mathrm{~d} s, \mathrm{~d} z)
$$

From (5) and (11),we have

$$
\begin{aligned}
& \mathrm{d} \Lambda_{t_{n}}^{t}=\Lambda_{t_{n}}^{t}\left[\int_{\mathcal{E}} \rho(e) g(t) \mathrm{d} \tilde{N}_{t}+h(t) \mathrm{d} W_{t}\right], \\
& \mathrm{d} y_{t}=-\left[r\left(t, x_{t}, y_{t}\right)+h(t) z_{t}+g(t) \Gamma_{t}\right] \mathrm{d} t+z_{t} \mathrm{~d} W_{t}+\int_{\mathcal{E}} U_{t} \mathrm{~d} \tilde{N}_{t}, \\
& \mathrm{~d} \Lambda_{t_{n}}^{t} y_{t}=\Lambda_{t_{n}}^{t} \mathrm{~d} y_{t}+y_{t} \mathrm{~d} \Lambda_{t_{n}}^{t}+\mathrm{d} \Lambda_{t_{n}}^{t} \mathrm{~d} y_{t} .
\end{aligned}
$$

From (12), (13) and (14), by applying Itô formula to $\Lambda_{t_{n}}^{t} y_{t}$, we obtain the equation

$$
\mathrm{d} \Lambda_{t_{n}}^{t} y_{t}=-\Lambda_{t_{n}}^{t} r\left(t, x_{t}, y_{t}\right) \mathrm{d} t+\Lambda_{t_{n}}^{t}\left[z_{t}+h(t) y_{t}\right] \mathrm{d} W_{t}+\Lambda_{t_{n}}^{t} \int_{\mathcal{E}}\left[U_{t}+y_{t} \rho(e) g(t)\right] \mathrm{d} \tilde{N}_{t} .
$$

From (15), it is easy to obtain that for $0 \leq n \leq N-1$,

$$
\begin{aligned}
y_{t_{n}}^{t_{n}, x^{n}}= & \Lambda_{t_{n}}^{t_{n+1}} y_{t_{n+1}, x^{n}}^{t_{n}}+\int_{t_{n}}^{t_{n+1}} \Lambda_{t_{n}}^{s} r\left(s, x_{s}^{t_{n}, x^{n}}, y_{s}^{t_{n}, x^{n}}\right) \mathrm{d} s-\int_{t_{n}}^{t_{n+1}} \Lambda_{t_{n}}^{s}\left[z_{s}^{t_{n}, x^{n}}+h(s) y_{s}^{t_{n}, x^{n}}\right] \mathrm{d} W_{s} \\
& -\int_{t_{n}}^{t_{n+1}} \int_{\mathcal{E}} \Lambda_{t_{n}}^{s}\left[U_{s}^{t_{n}, x^{n}}+y_{s}^{t_{n}, x^{n}} \rho(e) g(s)\right] \mathrm{d} \tilde{N}_{s},
\end{aligned}
$$

Taking the conditional mathematical expectation $\mathbb{E}_{t_{n}}^{x}[\cdot]$ on both side of the obtained equation, and by the nature of the conditional mathematical expectation,we deduce 


$$
y_{t_{n}}^{t_{n}, x^{n}}=\mathbb{E}_{t_{n}}^{x^{n}}\left[\Lambda_{t_{n}}^{t_{n+1}} y_{t_{n+1}}^{t_{n}, x^{n}}\right]+\int_{t_{n}}^{t_{n+1}} \mathbb{E}_{t_{n}}^{x^{n}}\left[\Lambda_{t_{n}}^{s} r\left(s, x_{s}^{t_{n}, x^{n}}, y_{s}^{t_{n}, x^{n}}\right)\right] \mathrm{d} s .
$$

Based on (17), we have

$$
y_{t_{n}}^{t_{n}, x^{n}}=\mathbb{E}_{t_{n}}^{x^{n}}\left[\Lambda_{t_{n}}^{t_{n+1}} y_{t_{n+1}}^{t_{n+1}, x^{n+1}}\right]+\frac{1}{2} \Delta t_{n}\left(r\left(t_{n}, x^{n}, y_{t_{n}}^{t_{n}, x^{n}}\right)+\mathbb{E}_{t_{n}}^{x^{n}}\left[\Lambda_{t_{n}}^{t_{n+1}} r\left(t_{n+1}, x^{n+1}, y_{t_{n+1}}^{t_{n+1}, x^{n+1}}\right)\right]\right)+\sum_{j=1}^{2} R_{y j}^{n},
$$

where

$$
R_{y_{1}}^{n}=\int_{t_{n}}^{t_{n+1}} \mathbb{E}_{t_{n}}^{x^{n}}\left[\Lambda_{t_{n}}^{t} r_{t}^{t_{n}, x^{n}}\right] \mathrm{d} t-\frac{1}{2} \Delta t_{n} \mathbb{E}_{t_{n}}^{x^{n}}\left[\Lambda_{t_{n}}^{t_{n+1}} r_{n_{n+1}}^{t_{n}, x^{n}}\right]-\frac{1}{2} \Delta t_{n} r_{t_{n}}^{t_{n}, x^{n}},
$$

and

$$
R_{y_{2}}^{n}=\mathbb{E}_{t_{n}}^{x^{n}}\left[\Lambda_{t_{n}}^{t_{n+1}}\left(y_{t_{n+1}}^{t_{n}, x^{n}}-y_{t_{n+1}}^{t_{n+1}, x^{n+1}}\right)\right]+\frac{1}{2} \Delta t_{n} \mathbb{E}_{t_{n}}^{x^{n}}\left[\Lambda_{t_{n}}^{t_{n+1}}\left(r_{t_{n+1}}^{t_{n}, x^{n}}-r_{t_{n+1}}^{t_{n+1}, x^{n+1}}\right)\right] .
$$

According to Lemma 1, the equality $\mathbb{E}_{t_{n}}^{x^{n}}\left[\Lambda_{t_{n}}^{t_{n+1}} y_{t_{n+1}}^{t_{n}, x^{n}}\right]=\tilde{\mathbb{E}}_{t_{n}}^{x^{n}}\left[y_{t_{n+1}}^{t_{n}, x^{n}}\right]$, we have

$$
y_{t_{n}}^{t_{n}, x^{n}}=\tilde{\mathbb{E}}_{t_{n}}^{x^{n}}\left[y_{t_{n+1}}^{t_{n}, x^{n}}\right]+\frac{1}{2} \Delta t_{n} r\left(t_{n}, x^{n}, y_{t_{n}}^{t_{n}, x^{n}}\right)+\frac{1}{2} \Delta t_{n} \tilde{\mathbb{E}}_{t_{n}}^{x^{n}}\left[r\left(t_{n+1}, x^{n+1}, y_{t_{n+1}}^{t_{n+1}, x^{n+1}}\right)\right]+\sum_{j=1}^{2} R_{y j}^{n} \text {, }
$$

Let $\Delta W_{t}=W_{t}-W_{t_{n}}$ for $t_{n} \leq t \leq t_{n+1}$. Then $\Delta W_{t}$ is a standard Brownian motion with mean zero and variance $t-t_{n}$. Now multiply (11) by $\Delta W_{t}$, taking the conditional mathematical expectation $\mathbb{E}_{t_{n}}^{x^{n}}[\cdot]$ on both sides of the obtained equation, and using the Itô isometric formula, we deduce

$$
0=\int_{t_{n}}^{t_{n+1}} \mathbb{E}_{t_{n}}^{x^{n}}\left[f_{s}^{t_{n}, x^{n}} \Delta W_{t_{n+1}}\right] \mathrm{d} s+\mathbb{E}_{t_{n}}^{x^{n}}\left[y_{t_{n+1}}^{t_{n}, x^{n}} \Delta W_{t_{n+1}}\right]-\int_{t_{n}}^{t_{n+1}} \mathbb{E}_{t_{n}}^{x^{n}}\left[z_{s}^{t_{n}, x^{n}}\right] \mathrm{d} s .
$$

From (22) we have,

$$
0=\mathbb{E}_{t_{n}}^{x^{n}}\left[y_{t_{n+1}}^{t_{n+1}, x^{n+1}} \Delta W_{t_{n+1}}\right]-\Delta t_{n} z_{t_{n}}^{t_{n}, x^{n}}+\sum_{j=1}^{2} R_{z j}^{n}
$$

where

$$
\begin{aligned}
& R_{z 1}^{n}=\int_{t_{n}}^{t_{n+1}} \mathbb{E}_{t_{n}}^{x^{n}}\left[f_{s}^{t_{n}, x^{n}} \Delta W_{t_{n+1}}\right] \mathrm{d} s-\int_{t_{n}}^{t_{n+1}}\left[\mathbb{E}_{t_{n}}^{x^{n}}\left[z_{s}^{t_{n}, x^{n}}\right]-z_{t_{n}}^{t_{n}, x^{n}}\right] \mathrm{d} s, \\
& R_{z 2}^{n}=\mathbb{E}_{t_{n}}^{x^{n}}\left[\left(y_{t_{n+1}}^{t_{n}, x^{n}}-y_{t_{n+1}}^{t_{n+1}, x^{n+1}}\right) \Delta W_{t_{n+1}}\right] .
\end{aligned}
$$

Let $\Delta \tilde{N}_{t_{n+1}}=\Delta N_{t_{n+1}}-\lambda \Delta t_{n}$, similarly multiplying (11) by $\Delta \tilde{N}_{t_{n+1}}$ yields

$$
0=\int_{t_{n}}^{t_{n+1}} \mathbb{E}_{t_{n}}^{x^{n}}\left[f_{s}^{t_{n}, x^{n}} \Delta \tilde{N}_{t_{n+1}}\right] \mathrm{d} s+\mathbb{E}_{t_{n}}^{x^{n}}\left[y_{t_{n+1}}^{t_{n}, x^{n}} \Delta \tilde{N}_{t_{n+1}}\right]-\int_{t_{n}}^{t_{n+1}} \mathbb{E}_{t_{n}}^{x^{n}}\left[\Gamma_{s}^{t_{n}, x^{n}}\right] \mathrm{d} s .
$$

From (25) we have,

$$
0=\mathbb{E}_{t_{n}}^{x^{n}}\left[y_{t_{n+1}}^{t_{n+1}, x^{n+1}} \Delta \tilde{N}_{t_{n+1}}\right]-\Delta t_{n} \Gamma_{t_{n}}^{t_{n}, x^{n}}+\sum_{j=1}^{2} R_{\Gamma j}^{n}
$$

where

$$
\begin{aligned}
R_{\Gamma 1}^{n} & =\int_{t_{n}}^{t_{n+1}} \mathbb{E}_{t_{n}}^{x^{n}}\left[f_{s}^{t_{n}, x^{n}} \Delta \tilde{N}_{t_{n+1}}\right] \mathrm{d} s-\int_{t_{n}}^{t_{n+1}}\left[\mathbb{E}_{t_{n}}^{x^{n}}\left[\Gamma_{s}^{t_{n}, x^{n}}\right]-\Gamma_{t_{n}}^{t_{n}, x^{n}}\right] \mathrm{d} s, \\
R_{\Gamma 2}^{n} & =\mathbb{E}_{t_{n}}^{x^{n}}\left[\left(y_{t_{n+1}}^{t_{n}, x^{n}}-y_{t_{n+1}}^{t_{n+1}, x^{n+1}}\right) \Delta \tilde{N}_{t_{n+1}}\right] .
\end{aligned}
$$

Based on (21), (23) and (26), for solving the BSDE (1) we propose the following scheme.

Scheme 1. Given $\left(x^{0}, y^{N}, z^{N}, \Gamma^{N}\right)$, solve $\left(x^{n+1}, y^{n}, z^{n}, \Gamma^{n}\right)$ backwardly by 


$$
\left\{\begin{array}{l}
x^{n+1}=x^{n}+\sum_{\alpha \in T_{2}} I_{\alpha}\left[f_{\alpha}\left(t_{n}, x^{n}, y^{n}\right)\right]_{t_{n}, t_{n+1}}, \\
y^{n}=\tilde{\mathbb{E}}_{t_{n}}^{x^{n}}\left[y^{n+1}\right]+\frac{1}{2} \Delta t_{n} f\left(t_{n}, x^{n}, y^{n}\right)+\frac{1}{2} \Delta t_{n} \tilde{\mathbb{E}}_{t_{n}}^{x^{n}}\left[f\left(t_{n+1}, x^{n+1}, y^{n+1}\right)\right] \\
\Delta t_{n} z^{n}=\mathbb{E}_{t_{n}}^{x^{n}}\left[y^{n+1} \Delta W_{t_{n+1}}\right] \\
\Delta t_{n} \Gamma^{n}=\mathbb{E}_{t_{n}}^{x^{n}}\left[y^{n+1} \Delta \tilde{N}_{t_{n+1}}\right] .
\end{array}\right.
$$

\section{Error Estimates}

In this section, we will give the error estimates of Scheme 1 proposed in Section 3. Now we introduce the error $e_{y}^{n}=y_{t_{n}}^{t_{n}, x^{n}}-y^{n}, \quad e_{z}^{n}=z_{t_{n}}^{t_{n}, x^{n}}-z^{n}$ and $e_{\Gamma}^{n}=\Gamma_{t_{n}}^{t_{n}, x^{n}}-\Gamma^{n}$ in $L^{p}$ norm, where $\left(x_{t}^{t_{n}, x^{n}}, y_{t}^{t_{n}, x^{n}}, z_{t}^{t_{n}, x^{n}}, \Gamma_{t}^{t_{n}, x^{n}}\right)$ is the solution of the FBSDEs (1), and $\left(x^{n+1}, y^{n}, z^{n}, \Gamma^{n}\right)$ is the solution of Scheme 1. For the sake of simplicity, we only consider one-dimensional BSDEs (i.e., $m=d=1$ ). However, all error estimate that we obtain in the sequel also hold for general multidimensional BSDEs. In our error analysis, we will use a constraint on the time partition step $\Delta t_{n}$ :

$$
\frac{\max _{0 \leq n \leq N-1} \Delta t_{n}}{\min _{0 \leq n \leq N-1} \Delta t_{n}} \leq c_{0} .
$$

Let us introduce the following Lemma, its proof can be found in the reference [2].

Lemma 2. Let $R_{y j}^{n}, R_{z j}^{n}$ and $R_{\Gamma j}^{n}(j=1,2)$ be the truncation errors defined in (21), (23) and (26), respectively. It holds that

$$
\begin{aligned}
& \max _{0 \leq n \leq N-1}\left\{\left|R_{y 1}^{n}\right|,\left|R_{z 1}^{n}\right|,\left|R_{\Gamma 1}^{n}\right|\right\} \leq C(\Delta t)^{3}, \\
& \max _{0 \leq n \leq N-1}\left\{\left|R_{y 2}^{n}\right|,\left|R_{z 2}^{n}\right|,\left|R_{\Gamma 2}^{n}\right|\right\} \leq C(\Delta t)^{3} .
\end{aligned}
$$

Here $C$ is a positive constant depending on $T$. We first give the error estimate for $y_{t_{n}}^{t_{n}, x^{n}}-y^{n}$ in the following theorem.

Theorem 1. Let $\left(x_{t}^{t_{n}, x^{n}}, y_{t}^{t_{n}, x^{n}}, z_{t}^{t_{n}, x^{n}}, \Gamma_{t}^{t_{n}, x^{n}}\right)_{0 \leq t \leq T}(0 \leq n \leq N-1)$ be the solution of the FBSDE (1) and $\left(x^{n+1}, y^{n}, z^{n}, \Gamma^{n}\right)$ be the solution of Scheme 1 . Assume $y^{N}=\varphi\left(X^{N}\right)$. Then for sufficiently small time step $\Delta t_{n}$, we have

$$
\max _{0 \leq n \leq N} \mathbb{E}\left[\left|y_{t_{n}}^{t_{n}, x^{n}}-y^{n}\right|^{p}\right] \leq C h^{2 p}
$$

for $1 \leq p \leq \infty$, where $C$ is a constant depending on $T$.

Proof. Let $e_{y}^{n}=y_{t_{n}}^{t_{n}, x^{n}}-y^{n}$. Subtracting (29) from (21) to get

$$
e_{y}^{n}=\tilde{\mathbb{E}}_{t_{n}}^{x^{n}}\left[y_{t_{n+1}}^{t_{n}, x^{n}}-y^{n+1}\right]+\frac{1}{2} \Delta t_{n}\left(e_{f}^{n}+\tilde{\mathbb{E}}_{t_{n}}^{x^{n}}\left[e_{f}^{n+1}\right]\right)+\sum_{j=1}^{2} R_{y j}^{n} .
$$

Under the conditions of the theorem and by Lemma 2, we deduce that,

$$
\begin{aligned}
e_{y}^{n} & \leq \tilde{\mathbb{E}}_{t_{n}}^{x^{n}}\left[e_{y}^{n+1}\right]+\frac{1}{2} \Delta t_{n} L\left|e_{y}^{n}\right|+\frac{1}{2} \Delta t_{n} L \tilde{\mathbb{E}}_{t_{n}}^{x^{n}}\left[\left|e_{y}^{n+1}\right|\right]+C\left(\Delta t_{n}\right)^{3} \\
& \leq\left(1+C \Delta t_{n}\right) \tilde{\mathbb{E}}_{t_{n} x^{n}}^{x^{n}}\left[\left|e_{y}^{n+1}\right|\right]+C \Delta t_{n}\left|e_{y}^{n}\right|+C\left(\Delta t_{n}\right)^{3},
\end{aligned}
$$

where $L$ is the Lipschitz constant of $f(t, x, y)$ with respect to $y$. Applying the inequality $(a+b)^{p} \leq a^{p}\left(1+\epsilon\left(2^{p-1}\right)\right)+b^{p}\left(1+\frac{2^{p-1}-1}{\epsilon^{p-1}}\right)$ for $0 \leq \epsilon \leq 1$ with $a=\left(1+C \Delta t_{n}\right) \tilde{\mathbb{E}}_{t_{n}}^{x^{n}}\left[\left|e_{y}^{n+1}\right|\right]$, 
$b=C \Delta t_{n}\left|e_{y}^{n}\right|+C\left(\Delta t_{n}\right)^{3}$, and $\epsilon=\Delta t_{n}$, we deduce,

$$
\begin{aligned}
\left|e_{y}^{n}\right|^{p} & \leq\left(\left(1+C \Delta t_{n}\right) \tilde{\mathbb{E}}_{t_{n}}^{x^{n}}\left[\left|e_{y}^{n+1}\right|\right]\right)^{p}\left(1+\Delta t_{n}\left(2^{p-1}-1\right)\right)+\left(C \Delta t_{n}\left|e_{y}^{n}\right|+C\left(\Delta t_{n}\right)^{3}\right)^{p}\left(1+\frac{2^{p-1}-1}{\left(\Delta t_{n}\right)^{p-1}}\right) \\
& \leq\left(1+C \Delta t_{n}\right)^{p+1} \tilde{\mathbb{E}}_{t_{n}}^{x^{n}}\left[\left|e_{y}^{n+1}\right|^{p}\right]+\left(C \Delta t_{n}\right)^{p}\left[\left|e_{y}^{n}\right|+\left(\Delta t_{n}\right)^{2}\right]^{p}\left(1+\frac{C}{\left(\Delta t_{n}\right)^{p-1}}\right),
\end{aligned}
$$

which by the inequality $(a+b)^{p} \leq 2^{p-1}\left(a^{p}+b^{p}\right)$ gives

$$
\left|e_{y}^{n}\right|^{p} \leq\left(1+C \Delta t_{n}\right) \tilde{\mathbb{E}}_{t_{n}}^{x^{n}}\left[\left|e_{y}^{n+1}\right|^{p}\right]+C \Delta t_{n}\left[\left|e_{y}^{n}\right|^{p}\right]+C\left(\Delta t_{n}\right)^{2 p+1} .
$$

Taking the mathematical expectation on both sides of (35), for sufficiently small $\Delta t_{n}$ we have

$$
\begin{aligned}
\left|e_{y}^{n}\right|^{p} & \leq \frac{(1+C h) \tilde{\mathbb{E}}_{t_{n}}^{x^{n}}\left[\left|e_{y}^{n+1}\right|^{p}\right]}{(1-C h)}+\frac{C h^{2 p+1}}{(1-C h)} \\
& \leq\left(\frac{1+C h}{1-C h}\right)^{N-n} \tilde{\mathbb{E}}_{t_{n}}^{x^{n}}\left[\left|e_{y}^{N}\right|^{p}\right]+\frac{C h^{2 p+1}}{1-C h} \sum_{i=0}^{N-n-1}\left(\frac{1+C h}{1-C h}\right)^{i},
\end{aligned}
$$

for $n=N-1, \cdots, 0$. The terminal condition $\mathbb{E}\left[\left|e_{y}^{N}\right|^{p}\right]=\mathbb{E}\left[\left|y_{T}-y^{N}\right|^{p}\right] \leq C\left(\Delta t_{n}\right)^{2 p}$, the time step constraint (28) and the inequality

$$
\frac{C h^{2 p+1}}{1-C h} \sum_{i=0}^{N-n-1}\left(\frac{1+C h}{1-C h}\right)^{i} \leq \frac{h^{2 p}}{2}\left(e^{2 c_{0} C T}-1\right)
$$

lead to $\mathbb{E}\left[\left|e_{y}^{n}\right|^{p}\right] \leq C h^{2 p}$ for $n=N-1, \cdots, 0$. The proof is completed.

Then we turn to estimating the error $e_{z}^{n}=z_{t_{n}}^{t_{n}, x^{n}}-z^{n}$.

Theorem 2. Let $\left(x_{t}^{t_{n}, x^{n}}, y_{t}^{t_{n}, x^{n}}, z_{t}^{t_{n}, x^{n}}, \Gamma_{t}^{t_{n}, x^{n}}\right)$ be the solution of the FBSDE(1) and $\left(x^{n+1}, y^{n}, z^{n}, \Gamma^{n}\right)$ be the solution of Scheme 1. Assume $\mathbb{E}\left[\left|e_{y}^{N}\right|^{p}\right]=\mathbb{E}\left[\left|y_{T}-y^{N}\right|^{p}\right] \leq C\left(\Delta t_{n}\right)^{2 p}$. Then for sufficiently small time step $\Delta t_{n}$, we have

$$
\max _{0 \leq n \leq N} \mathbb{E}\left[\left|z_{t_{n}}^{t_{n}, x^{n}}-z^{n}\right|^{p}\right] \leq C\left(\Delta t_{n}\right)^{p}
$$

for $1 \leq p \leq \infty$, where $C$ is a constant depending on $T$.

Proof. Let $e_{z}^{n}=z_{t_{n}}^{t_{n}, x^{n}}-z^{n}$. From (19) and (25), we get

$$
\Delta t_{n} e_{z}^{n}=\mathbb{E}_{t_{n}}^{x^{n}}\left[e_{y}^{n+1} \Delta W_{t_{n+1}}\right]+\sum_{j=1}^{2} R_{z j}^{n} .
$$

By Lemma 2, the inequality $(a+b)^{p} \leq 2^{p-1}\left(a^{p}+b^{p}\right)$ and Hölder's inequality, we deduce

$$
\begin{aligned}
\left(\Delta t_{n}\left|e_{z}^{n}\right|\right)^{p} & \leq 2^{p-1}\left|\mathbb{E}_{t_{n}}^{x^{n}}\left[e_{y}^{n+1} \Delta W_{t_{n+1}}\right]\right|^{p}+2^{p-1} \sum_{j=1}^{2}\left|R_{z j}^{n}\right|^{p} \\
& \leq C_{p}\left(\mathbb{E}_{t_{n}}^{x^{n}}\left[\left|e_{y}^{n+1}\right|^{2}\right]^{\frac{p}{2}}\left(\Delta t_{n}\right)^{\frac{p}{2}}+\left(\Delta t_{n}\right)^{2 p}\right) \\
& \leq C_{p}\left(\mathbb{E}_{t_{n}}^{x^{n}}\left[\left|e_{y}^{n+1}\right|^{2}\right]^{\frac{p}{2}} h^{\frac{p}{2}}+h^{2 p}\right)
\end{aligned}
$$

where $C_{p}$ is a positive number which depends on $p$ and the constant $C$ in Lemma 2. Taking the mathematical 
expectation on both sides of the Equation (38) gives

$$
\left(\Delta t_{n}\right)^{p} \mathbb{E}\left[\left|e_{z}^{n}\right|^{p}\right] \leq C_{p}\left(\mathbb{E}\left[\left|e_{y}^{n+1}\right|^{2}\right]^{\frac{p}{2}} h^{\frac{p}{2}}+h^{2 p}\right),
$$

by using Theorem 1 and constraint (28), leads to $\mathbb{E}\left[\left|e_{z}^{n}\right|^{p}\right] \leq C h^{p}$ for $n=N-1, \cdots, 1,0$. The proof is completed.

At last, we estimate the error $e_{\Gamma}^{n}=\Gamma_{t_{n}}^{t_{n}, x^{n}}-\Gamma^{n}$.

Theorem 3. Let $\left(x_{t}^{t_{n}, x^{n}}, y_{t_{n}}^{t_{n}, x^{n}}, z_{t_{n}}^{t_{n}, x^{n}}, \Gamma_{t_{n}}^{t_{n}, x^{n}}\right)$ be the solution of the FBSDE(1) and $\left(x^{n+1}, y^{n}, z^{n}, \Gamma^{n}\right)$ be the solution of Scheme 1. Assume $\mathbb{E}\left[\left|e_{y}^{N}\right|^{p}\right]=\mathbb{E}\left[\left|y_{T}-y^{N}\right|^{p}\right] \leq C\left(\Delta t_{n}\right)^{2 p}$. Then for sufficiently small time step $\Delta t_{n}$, we have

$$
\max _{0 \leq n \leq N} \mathbb{E}\left[\left|\Gamma_{t_{n}}^{t_{n}, x^{n}}-\Gamma^{n}\right|^{p}\right] \leq C\left(\Delta t_{n}\right)^{p}
$$

for $1 \leq p \leq \infty$, where $C$ is a constant depending on $T$.

Proof. Let $e_{\Gamma}^{n}=\Gamma_{t_{n}}^{t_{n}, x^{n}}-\Gamma^{n}$. From (26) and (31),we get

$$
\Delta t_{n} e_{\Gamma}^{n}=\mathbb{E}_{t_{n}}^{x^{n}}\left[e_{y}^{n+1} \Delta \tilde{N}_{t_{n+1}}\right]+\sum_{j=1}^{2} R_{\Gamma j}^{n} .
$$

By Lemma 2, the inequality $(a+b)^{p} \leq 2^{p-1}\left(a^{p}+b^{p}\right)$ and Hölder's inequality, we deduce

$$
\begin{aligned}
\left(\Delta t_{n}\left|e_{\Gamma}^{n}\right|\right)^{p} & \leq 2^{p-1}\left|\mathbb{E}_{t_{n}}^{x^{n}}\left[e_{y}^{n+1} \Delta \tilde{N}_{t_{n+1}}\right]\right|^{p}+2^{p-1}\left|R_{\Gamma}^{n}\right|^{p} \\
& \leq C_{p}\left(\mathbb{E}_{t_{n}}^{x^{n}}\left[\left|e_{y}^{n+1}\right|^{2}\right]^{\frac{p}{2}}\left(\Delta t_{n}\right)^{\frac{p}{2}}+\left(\Delta t_{n}\right)^{2 p}\right) \\
& \leq C_{p}\left(\mathbb{E}_{t_{n}}^{x^{n}}\left[\left|e_{y}^{n+1}\right|^{2}\right]^{\frac{p}{2}} h^{\frac{p}{2}}+h^{2 p}\right),
\end{aligned}
$$

where $C_{p}$ is a positive number which depends on $p$ and the constant $C$ in Lemma 2. Taking the mathematical expectation on both sides of the Equation (42) gives

$$
\left(\Delta t_{n}\right)^{p} \mathbb{E}\left[\left|e_{\Gamma}^{n}\right|^{p}\right] \leq C_{p}\left(\mathbb{E}\left[\left|e_{y}^{n+1}\right|^{2}\right]^{\frac{p}{2}} h^{\frac{p}{2}}+h^{2 p}\right),
$$

by using Theorem 1 and constraint (28), leads to $\mathbb{E}\left[\left|e_{\Gamma}^{n}\right|^{p}\right] \leq C h^{p}$ for $n=N-1, \cdots, 1,0$. The proof is completed.

\section{References}

[1] Pardoux, E. and Peng, S. (1990) Adapted Solution of a Backward Stochastic Differntial Equation. Systems \& Control Letters, 14, 55-61. http://dx.doi.org/10.1016/0167-6911(90)90082-6

[2] Li, Y. and Zhao, W. (2010) $\mathrm{L}^{\mathrm{p}}$-Error Estimates for Numerical Schemes for Solving Certain Kinds of Backward Stochastic Differential Equations. Statistics and Probability Letters, 80, 21-22, 1612-1617.

[3] Zhao, W., Chen, L. and Peng, S. (2006) A New Kind of Accurate Numerical Method for Backward Stochastic Differential Equations. SIAM Journal on Scientific Computing, 28, 1563-1581. http://dx.doi.org/10.1137/05063341X

[4] Tang, S. and Li, X. (1994) Necessary Conditions for Optimal Control of Stochastic Systems with Random Jumps. SIAM Journal on Control and Optimization, 32, 1447-1475. http://dx.doi.org/10.1137/S0363012992233858 


\section{Submit or recommend next manuscript to SCIRP and we will provide best service for you:}

Accepting pre-submission inquiries through Email, Facebook, LinkedIn, Twitter, etc.

A wide selection of journals (inclusive of 9 subjects, more than 200 journals)

Providing 24-hour high-quality service

User-friendly online submission system

Fair and swift peer-review system

Efficient typesetting and proofreading procedure

Display of the result of downloads and visits, as well as the number of cited articles

Maximum dissemination of your research work

Submit your manuscript at: http://papersubmission.scirp.org/ 